International Journal of Engineering, Science and Technology

Vol. 1, No. 1, 2009, pp. 216-227
INTERNATIONAL JOURNAL OF

ENGINEERING, SCIENCE AND

TECHNOLOGY

www.ijest-ng.com

(C) 2009 MultiCraft Limited. All rights reserved

\title{
Response of multiphase magneto-electro-elastic sensors under harmonic mechanical loading
}

\author{
B. Biju, N. Ganesan and K.Shankar*
}

Machine Design Section, Department of Mechanical Engineering, Indian Institute of Technology, Madras, Chennai 600 036, India E-mails:(skris@iitm.ac.in (K. Shankar),Tel.: +91 44 22574701, "Corresponding author); bbiju@rediffmail.com (B. Biju)

\begin{abstract}
The finite element formulation for coupled magneto-electro-elastic sensor bonded to a mild steel beam with plane stress assumption is presented in this paper. The beam is subjected to harmonic excitation with a point load at tip and a uniformly distributed load along the bottom surface of the mild steel beam. Numerical results are presented for clamped free boundary condition for the first three modes of the structure. The sensor response is dominated by the first mode, but the third mode response become significant when the sensor is placed at the free end of the mild steel beam.
\end{abstract}

Keywords: Magneto-electro-elastic, sensor, harmonic response, finite element

\section{Introduction}

Smart material is a continuum whose mechanical constitutive response is coupled with non-mechanical phenomena. Magnetoelectro-elastic (MEE) materials belong to the family of smart materials; exhibit a significant coupling between mechanical, electric and magnetic fields. The composite consisting of piezoelectric phase shows a coupling between mechanical and electric field whereas the piezomagnetic phase shows the coupling between mechanical and magnetic field .In addition to this, a magnetoelectric coupling effect which is absent in the constituent phases is exhibited by these classes of magneto-electro-elastic materials. Due to exceptional nature of these materials to convert one form of energy into another, these materials are widely substituted in acoustic devices, medical ultrasonic imaging etc. Another identified area of application is as sensors and actuators. Response characteristics of MEE sensors under dynamic loading will help to determine the best operating conditions of MEE devices which in turn will lead to emerging areas for application of magneto-electro-elastic materials as sensors and actuators.

Fibrous composites consisting of piezomagnetic cobalt iron oxide $\mathrm{CoFe}_{2} \mathrm{O}_{4}$ matrix reinforced by piezoelectric barium titanate $\mathrm{BaTiO}_{3}$ fibers (Buchanan, 2004) are analysed for the sensory response. Both phases are traversely isotropic with the axis of symmetry oriented in the z-direction. Aboudi (2001) has employed a homogenization method assuming that the composites have a periodic structure, for predicting the effective moduli of magneto-electro-elastic composites. Ramirez et al. (2006) presented approximate solution to a free vibration problem of a two dimensional MEE laminate assuming perfect bonding between each interface. Bhangale et al. (2005) conducted free vibration studies on functionally graded MEE cylindrical shells and evaluated the influence of piezomagnetic and piezoelectric effect on structural frequency. Transient response of magneto-electro-elastic hollow cylinder to axisymmetric mechanical and electromagnetic loading was done by Hou et al. (2004). Transient responses of displacements, stresses, electric and magnetic potentials, electric displacements, magnetic induction are obtained by the study. Dai et al. (2006) have presented an analytical solution for magneto-thermo-electro-elastic transient response of a piezoelectric hollow cylinder placed in an axial magnetic field subjected to arbitrary thermal shock, mechanical load and transient electric excitation. Sirohi et al. (2000) investigated the piezoelectric strain sensor in which strain is measured in terms of charge developed by direct piezoelectric effect. Huang et al. (2000) examined the dynamic electromechanical response of piezoelectric sensors and actuators which are modeled as rectangular plate and the variation of electric potential, stresses and electric displacements across the thickness were evaluated. Pietrzakowski et al. (2008) dealt with active vibration control of rectangular plates containing piezoelectric sensor/actuator layers using velocity control feed back to suppress vibration. Galopin et al. (2008) has done finite 
element modeling of magnetoelectic sensors and the magnetoelectric effect stemming from piezoelectric and magnetosticive composite was studied. Daga et al. (2009) has studied the behavior of MEE sensors under transient mechanical loading.

This paper deals with response to harmonic mechanical loading of magneto-electro-elastic sensor, which has not been hither to address. The sensor is bonded on the top surface of a mild steel beam. Finite element method with plane stress assumption is used in the analysis. Dynamic response of the sensor is studied when the beam is subjected to clamped free boundary condition with the sensor placed at different positions on the top surface of the beam. A point load at the tip of the beam and a uniformly distributed load along the length of the beam in the negative z-direction are used for the numerical studies. These studies will be highly useful when we use MEE sensors and actuators for active vibration control of structures. Ansys 10.1 is used to validate the computer code developed for the study. Ansys cannot model fully coupled magneto-electro-elastic materials but it can model piezoelectric PZT-5 which is used to compare the results of the code.

\section{Constitutive equations}

The constitutive equations for the MEE medium relating stress $\sigma_{j}$, electric displacement $D_{l}$ and magnetic induction $B_{l}$ to strain $S_{k}$, electric field $E_{m}$ and magnetic field $H_{m}$, exhibiting linear coupling between magnetic, electric and elastic field can be written as (Daga et al., 2008)

$$
\begin{aligned}
& \sigma_{j}=C_{j k} S_{k}-e_{j m} E_{m}-q_{j m} H_{m} \\
& D_{l}=e_{l j} S_{k}+\varepsilon_{l m} E_{m}+m_{l m} H_{m} \\
& B_{l}=q_{l j} S_{k}+m_{l m} E_{m}+\mu_{l m} H_{m}
\end{aligned}
$$

where $C_{j k}, \varepsilon_{l m}$ and $\mu_{l m}$ are elastic, dielectric and magnetic permeability coefficients respectively and $e_{l j}, q_{l j}$ and $m_{l m}$ are the piezoelectric, piezomagnetic and magnetoelectric material coefficients. Here $j, k=1, \ldots, 6$ and $l, m=1, \ldots, 3$.

\section{Finite element modeling of magneto-electro-elastic beam}

The potential for MEE solid (Sunar et al., 2002) can be written as

$$
G=\frac{1}{2} S^{T} C S-\frac{1}{2} E^{T} \varepsilon E-\frac{1}{2} H^{T} \mu H-S e E-S q H-E m H
$$

In the present formulation body forces, free charge density and free current density are absent. In the absence of free charge density, Gauss law can be written as $\nabla \cdot D=0$ where $D$ is the electric displacement vector. The scalar electric potential $\phi$ is defined so that the electric field $E$ can be written as $E=-\nabla \phi$. Gauss law for magnetic field is written as $\nabla \cdot B=0$ where $B$ is the magnetic induction vector. The magnetic scalar potential $\psi$ which satisfies the above relation is defined so that the magnetic field $H$ can be written as $H=-\nabla \psi$.

The mild steel beam with sensor bonded to its top surface is modeled using four nodded rectangular plate element with 4 degrees of freedom per node i.e. displacement in axial direction $\left(u_{x}\right)$, displacement in transverse direction $\left(u_{z}\right)$, electric potential $(\phi)$ and magnetic potential $(\psi)$. Figure 1 schematically represents the finite element discretization of the mild steel beam with a magnetoelectro-elastic sensor bonded at the top surface using four nodded rectangular plate element.

The displacements $\{u\}=\left\{\begin{array}{ll}u_{x} & u_{z}\end{array}\right\}^{T}$, electric potential $(\phi)$ and magnetic potential $(\psi)$ within the element can be expressed in terms of suitable shape functions $N_{i}$ and the corresponding nodal quantities as given below.

$$
\{u\}=\left[N_{i}\right]\left\{u^{e}\right\} ; \phi=\left[N_{i}\right]\left\{\phi^{e}\right\} ; \psi=\left[N_{i}\right]\left\{\psi^{e}\right\} \text { where } \mathrm{i}=1, \ldots ., 4 \text {. }
$$




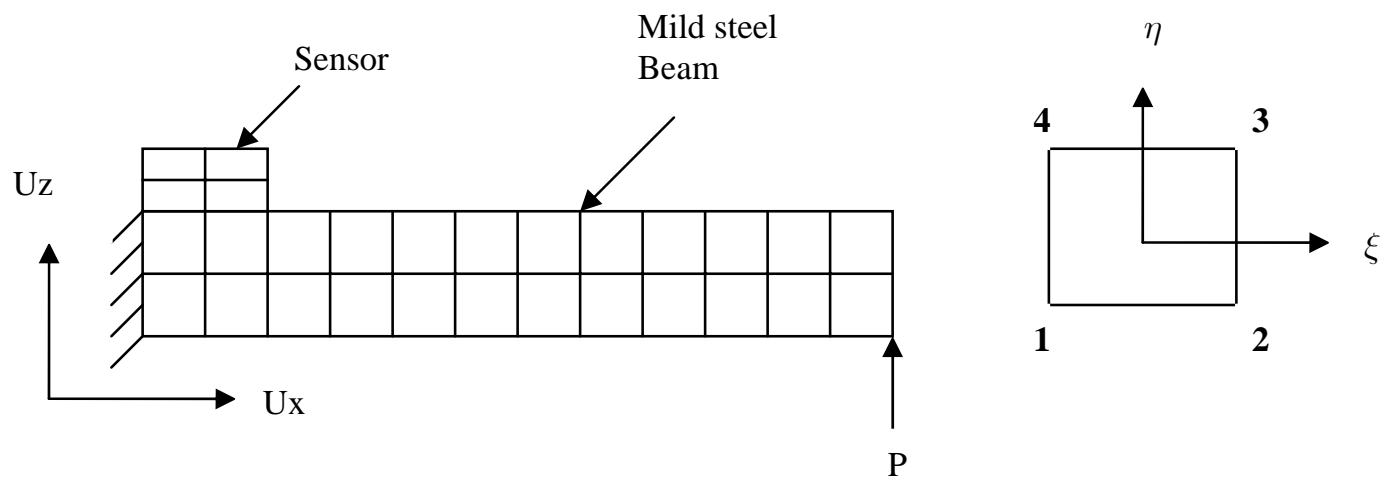

Figure 1. Finite Element discretization of the mild steel beam with sensor bonded at the clamped end.

For a plane stress problem the stress components $\sigma_{2}\left(\sigma_{y y}\right)=\sigma_{4}\left(\tau_{y z}\right)=\sigma_{6}\left(\tau_{x y}\right)=0$, electric displacement component $D_{2}\left(D_{y}\right)=0$ and magnetic induction component $B_{2}\left(B_{y}\right)=0$. The strain displacement relation for a 2D plane stress problem can be written as

$$
S_{1}=S_{x x}=\frac{\partial u_{x}}{\partial x} ; \quad S_{2}=S_{z z}=\frac{\partial u_{z}}{\partial z} ; \quad S_{5}=S_{x z}=\frac{\partial u_{x}}{\partial z}+\frac{\partial u_{z}}{\partial x}
$$

The relation of electric field to the electric potential can be expressed as

$$
E_{1}=E_{x}=\frac{\partial \phi}{\partial x} ; E_{3}=E_{z}=\frac{\partial \phi}{\partial z}
$$

The relation of magnetic field to the magnetic potential can be expressed as

$$
H_{1}=H_{x}=\frac{\partial \psi}{\partial x} ; H_{3}=H_{z}=\frac{\partial \psi}{\partial z}
$$

Jiang et al. (2004) have derived the reduced material constants under a plane stress assumption, which are used to evaluate the stiffness matrices. A finite element formulation for the fully coupled magneto-electro-elastic continuum can be expressed as

$$
\begin{aligned}
& {\left[M_{u u}\right]\{\ddot{u}\}+\left[K_{u u}\right]\{u\}+\left[K_{u \phi}\right]\{\phi\}+\left[K_{u \psi}\right]\{\psi\}=\left\{F e^{i \omega t}\right\}} \\
& {\left[K_{u \phi}\right]^{T}\{u\}-\left[K_{\phi \phi}\right]\{\phi\}-\left[K_{\phi \psi}\right]\{\psi\}=0} \\
& {\left[K_{u \psi}\right]^{T}\{u\}-\left[K_{\phi \psi}\right]^{T}\{\phi\}-\left[K_{\psi \psi}\right]\{\psi\}=0}
\end{aligned}
$$

where $F e^{i \omega t}$ is the harmonically applied mechanical load which is varying with the circular frequency $\omega$. Since no electric charge or current density is applied, the right hand side of equation $9 \mathrm{~b}$ and $9 \mathrm{c}$ is zero. Elemental mass and stiffness matrices corresponding to the above equations are derived as follows.

$$
\begin{aligned}
& {\left[K_{u u}\right]=\int_{v}\left[B_{u}\right]^{T}[C]\left[B_{u}\right] d V} \\
& {\left[K_{u \phi}\right]=\int_{v}\left[B_{u}\right]^{T}[e]\left[B_{\phi}\right] d V} \\
& {\left[K_{u \psi}\right]=\int_{v}\left[B_{u}\right]^{T}[q]\left[B_{\psi}\right] d V}
\end{aligned}
$$




$$
\begin{aligned}
& {\left[K_{\phi \phi}\right]=\int_{v}\left[B_{\phi}\right]^{T}[\varepsilon]\left[B_{\phi}\right] d V} \\
& {\left[K_{\psi \psi}\right]=\int_{v}\left[B_{\psi}\right]^{T}[\mu]\left[B_{\psi}\right] d V} \\
& {\left[K_{\phi \psi}\right]=\int_{v}\left[B_{\phi}\right]^{T}[m]\left[B_{\psi}\right] d V} \\
& {\left[M_{\text {uи }}\right]=\int_{v}[N]^{T}[\rho][N] d V}
\end{aligned}
$$

$\left[B_{u}\right],\left[B_{\phi}\right]$ and $\left[B_{\psi}\right]$ represent the shape function derivative matrices for strain-displacement, electric field-electric potential and magnetic field-magnetic potential respectively. Three point Gauss quadrature is used to evaluate the integrals.

Using standard condensation techniques, the electric potential and magnetic potential are eliminated from equation (9) which can be written as

$$
\left[M_{u u}\right]\{\ddot{u}\}+\left[K_{e q}\right]\{u\}=\left\{F e^{i \omega t}\right\}
$$

where $\left[K_{e q}\right]$ is the equivalent stiffness matrix.

$$
\left[K_{e q}\right]=\left[K_{u u}\right]+\left[K_{u \phi}\right]\left[K_{I I}\right]^{-1}\left[K_{I}\right]+\left[K_{u \psi}\right]\left[K_{I V}\right]^{-1}\left[K_{I I I}\right]
$$

The component matrices in equation 11 can be written as

$$
\begin{aligned}
& {\left[K_{I}\right]=\left[K_{u \phi}\right]^{T}-\left[K_{\phi \psi}\right]\left[K_{\psi \psi}\right]^{-1}\left[K_{u \psi}\right]^{T}} \\
& {\left[K_{I I}\right]=\left[K_{\phi \phi}\right]-\left[K_{\phi \psi}\right]\left[K_{\psi \psi}\right]^{-1}\left[K_{\phi \psi}\right]^{T}} \\
& {\left[K_{I I I}\right]=\left[K_{u \psi}\right]^{T}-\left[K_{\phi \psi}\right]^{T}\left[K_{\phi \phi}\right]^{-1}\left[K_{u \phi}\right]^{T}} \\
& {\left[K_{I V}\right]=\left[K_{\psi \psi}\right]-\left[K_{\phi \psi}\right]^{T}\left[K_{\phi \phi}\right]^{-1}\left[K_{\phi \psi}\right]}
\end{aligned}
$$

The Eigen vectors corresponding to $\phi$ and $\psi$ is given by

$$
\begin{aligned}
& \{\phi\}=\left[K_{I I}\right]^{-1}\left[K_{I}\right]\{u\} \\
& \{\psi\}=\left[K_{I V}\right]^{-1}\left[K_{I I I}\right]\{u\}
\end{aligned}
$$

\section{Validation}

A computer code has been developed to study the response of magneto-electro-elastic sensor bonded on the top surface of a cantilever beam while the beam is excited harmonically. Ansys 10.1 used for validation studies cannot model fully coupled magneto-electro-elastic materials but it can model piezoelectric materials. The present code is validated using piezoelectric material PZT-5 whose material properties (Chen et al., 2007) are given in Table 1. The dimensions of mild steel beam are as follows: length $=0.3 \mathrm{~m}$, thickness $=0.02 \mathrm{~m}$. The dimensions of the sensor patch are length $=0.015 \mathrm{~m}$, thickness $=0.002 \mathrm{~m}$. The PZT sensor is placed at the clamped end of the beam and a point load of $1 \mathrm{~N}$ is applied at the free end in the negative z-direction.

Finite element mesh for the mild steel beam and the sensor patch are $40 \times 2$ and $2 \times 2$ respectively. Full method is used as the solution technique and a constant damping ratio of 0.01 is used. Response is taken from that node of the sensor which shows maximum value of electric potential. The absolute value of transverse displacement $\left(u_{z}\right)$ and the electric potential $(\phi)$ corresponding to the above said node are obtained for the first two natural frequencies of the structure. Log scale plots of the responses using code and Ansys are shown in Figure 2(a) and 2(b), which are in good agreement. Hence the above computer code is used to study the response of magneto-electro-elastic sensors under harmonic mechanical loading. 
Table1. Material properties of PZT-5 and different volume fraction $v_{f}$ of multiphase magneto-electro-elastic $\mathrm{BaTiO}_{3}-\mathrm{CoFe}_{2} \mathrm{O}_{4}$ (Aboudi, 2001)

\begin{tabular}{|l|c|c|c|c|c|c|c|}
\hline & $0.0 v_{f}$ & $0.2 v_{f}$ & $0.4 v_{f}$ & $0.6 v_{f}$ & $0.8 v f$ & $1.0 v_{f}$ & PZT-5 \\
\hline Elastic constants & & & & & & & \\
\hline $\mathrm{C}_{11}=\mathrm{C}_{22}$ & 286 & 250 & 225 & 200 & 175 & 166 & 99.2 \\
\hline $\mathrm{C}_{12}$ & 173 & 146 & 125 & 110 & 100 & 77 & 54.01 \\
\hline $\mathrm{C}_{13}=\mathrm{C}_{23}$ & 170 & 145 & 125 & 110 & 100 & 78 & 50.77 \\
\hline $\mathrm{C}_{33}$ & 269.5 & 240 & 220 & 190 & 170 & 162 & 86.85 \\
\hline $\mathrm{C}_{44}=\mathrm{C}_{55}$ & 45.3 & 45 & 45 & 45 & 50 & 43 & 21.1 \\
\hline Piezoelectric constants & & & & & & & \\
\hline $\mathrm{e}_{31}=\mathrm{e}_{32}$ & 0 & -2 & -3.0 & -3.5 & -4.0 & -4.4 & -7.20 \\
\hline $\mathrm{e}_{33}$ & 0 & 4 & 7.0 & 11.0 & 14.0 & 18.6 & 15.11 \\
\hline $\mathrm{e}_{24}=\mathrm{e}_{15}$ & 0 & 0 & 0 & 0 & 0 & 11.6 & 12.32 \\
\hline Dielectric constants & & & & & & & \\
\hline$\varepsilon_{11}=\varepsilon_{22}$ & 0.08 & 0.33 & 0.8 & 0.9 & 1.0 & 11.2 & 1.53 \\
\hline$\varepsilon_{33}$ & 0.093 & 2.5 & 5.0 & 7.5 & 10 & 12.6 & 1.5 \\
\hline $\begin{array}{l}\text { Magnetic permeability } \\
\text { constants }\end{array}$ & & & & & & & \\
\hline$\mu_{11}=\mu_{22}$ & -5.9 & -3.9 & -2.5 & -1.5 & -0.8 & 0.05 & 0 \\
\hline$\mu_{33}$ & 1.57 & 1.33 & 1.0 & 0.75 & 0.5 & 0.1 & 0 \\
\hline Piezomagnetic constants & & & & & & & \\
\hline $\mathrm{q}_{31}=\mathrm{q}_{32}$ & 580 & 410 & 300 & 200 & 100 & 0 & 0 \\
\hline $\mathrm{q}_{33}$ & 700 & 550 & 380 & 260 & 120 & 0 & 0 \\
\hline $\mathrm{q}_{24}=\mathrm{q}_{15}$ & 560 & 340 & 220 & 180 & 80 & 0 & 0 \\
\hline Magnetoelectric constants & & & & & & & \\
\hline $\mathrm{m}_{11}=\mathrm{m}_{22}$ & 0 & 2.8 & 4.8 & 6.0 & 6.8 & 0 & 0 \\
\hline $\mathrm{m}_{33}$ & 0 & 2000 & 2750 & 2500 & 1500 & 0 & 0 \\
\hline Density & & & & & & \\
\hline$\rho$ & 5300 & 5400 & 5500 & 5600 & 5700 & 5800 & 7750 \\
\hline
\end{tabular}

$\mathrm{C}_{\mathrm{ij}}$ in $10^{9} \mathrm{~N} / \mathrm{m}^{2}, \mathrm{e}_{\mathrm{ij}}$ in $\mathrm{C} / \mathrm{m}^{2}, \varepsilon_{l k}$ in $10^{-9} \mathrm{C} / \mathrm{Vm}, \mathrm{q}_{\mathrm{ij}}$ in $\mathrm{N} / \mathrm{Am}, \mu_{l k}$ in $10^{-4} \mathrm{Ns}^{2} / \mathrm{C}^{2}, \mathrm{~m}_{\mathrm{lk}}$ in $10-{ }^{12} \mathrm{Ns} / \mathrm{VC}, \rho$ inkg $/ \mathrm{m}^{3}$

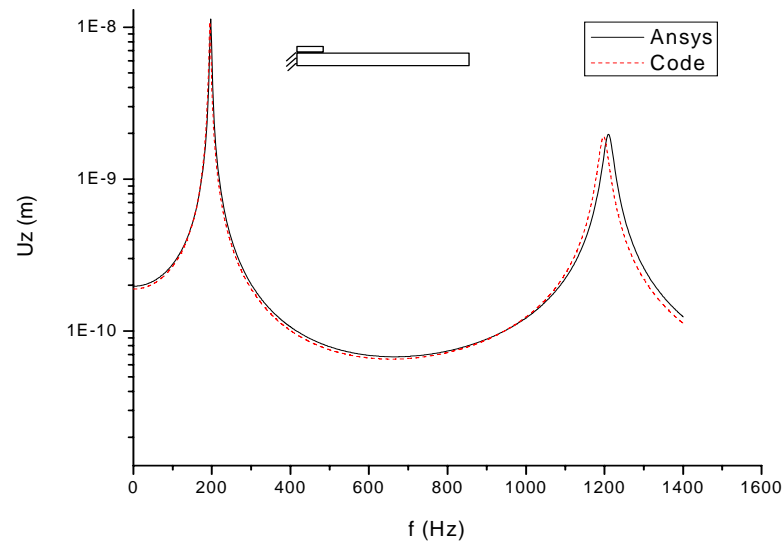

(a)

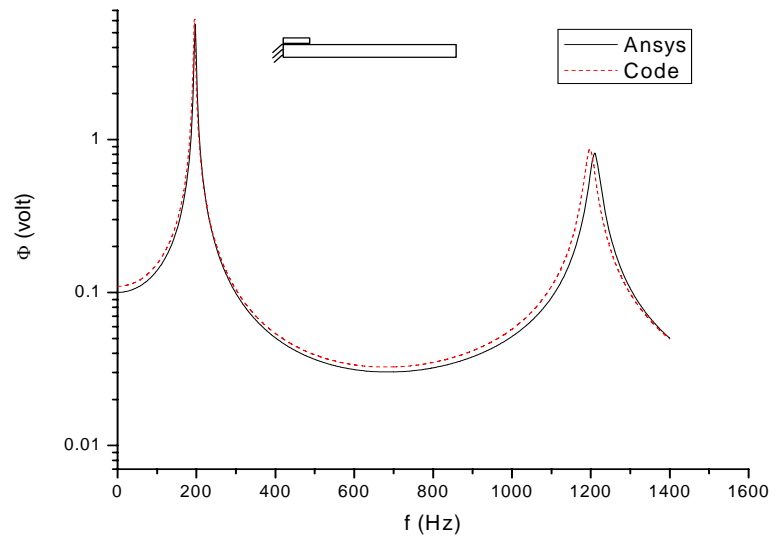

(b)

Figure 2 (a) Transverse displacement and (b) Electric potential $(\phi)$ at the top surface of the PZT-5 sensor 


\section{Results and discussion}

Response of magneto-electro-elastic sensors bonded on the top surface of a cantilever beam with clamped-free boundary condition under harmonic mechanical loading is discussed below. Numerical results are presented for clamped free boundary condition for the first three modes of the structure.

Two types of loadings are used. (i) A point load of $1 \mathrm{~N}$ at the tip of the beam in the negative z-direction (ii) Uniformly distributed load of $1 \mathrm{~N}$ at the bottom surface of the beam in the negative z-direction. The location of the sensor is varied along the length of the beam i.e. at the clamped end, at the centre and at the tip of the beam. The harmonic response of the system is analysed with the base beam subjected to clamped-free boundary condition with $u_{x}=u_{z}=\phi=\psi=0$ at the clamped end. The electric potential $(\phi)$ at the interface between base beam and sensor is also assumed to be zero. The material properties of multiphase MEE material with volume fraction of $\mathrm{BaTiO}_{3}$ varying from 0.0 to 1.0 in a composite of $\mathrm{BaTiO}_{3}-\mathrm{CoFe}_{2} \mathrm{O}_{4}$ are listed in Table 1. Volume fraction of 0.0 represents pure $\mathrm{CoFe}_{2} \mathrm{O}_{4}$ and volume fraction of 1.0 represents pure $\mathrm{BaTiO}_{3}$. The material properties of mild steel beam are as follows: Young's modulus $(\mathrm{E})=210 \mathrm{GPa}$, Poisson ratio $(v)=0.3$, Magnetic permeability constant $(\mu)=2.51 \mathrm{e}-4$, density $(\rho)$ $=7800 \mathrm{~kg} / \mathrm{m}^{3}$. The sensor response during the first three modes for different volume fraction of the MEE composite is plotted graphically.

\subsection{A point load at the tip of the beam}

The beam is clamped at one end and response of the sensor is studied when the beam is subjected to a harmonic excitation of $1 \mathrm{~N}$ in the negative z-direction at the free end of the beam.

\subsubsection{Sensor patch located at the clamped end}

Figure 3 shows the transverse displacement $\left(u_{z}\right)$, electric potential $(\phi)$ and magnetic potential $(\psi)$ at the top surface of the MEE sensor when the sensor is located at the clamped end of the mild steel beam. The transverse displacement is found maximum at the first mode. It goes on increasing as the volume fraction increases attributing to the fact that the elastic constants decreases as the volume fraction increases. The electric potential $(\phi)$ is found maximum for $0.2 v_{f}$ of the composite and the electric potential decreases considerably at higher modes. Volume fraction 1.0 which corresponds to pure $\mathrm{BaTiO}_{3}$ also gives electric potential comparable to $0.2 v_{f}$. The magnetic potential $(\psi)$ is found maximum for $0.6 v_{f}$ of the composite and the magnetic potential also decreases at higher modes. Volume fraction 0.0 which corresponds to pure $\mathrm{CoFe}_{2} \mathrm{O}_{4}$ gives less comparable magnetic potential out put with $0.6 v_{f}$.

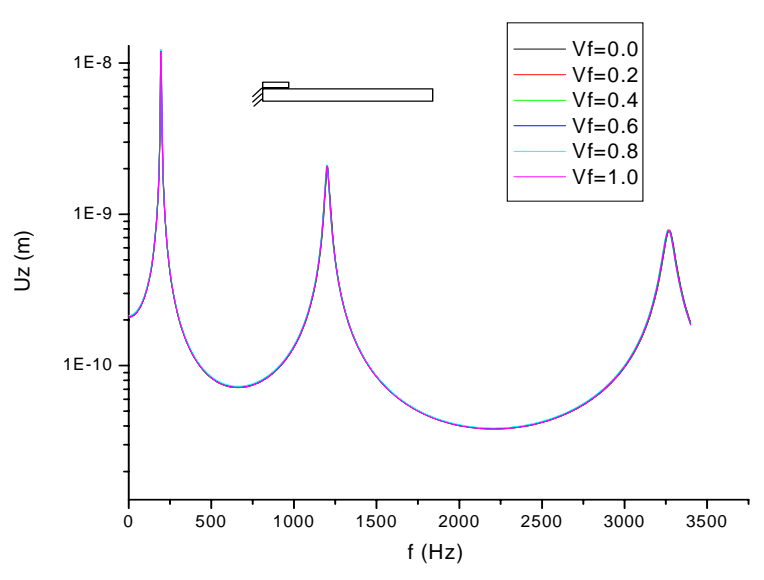

(a)

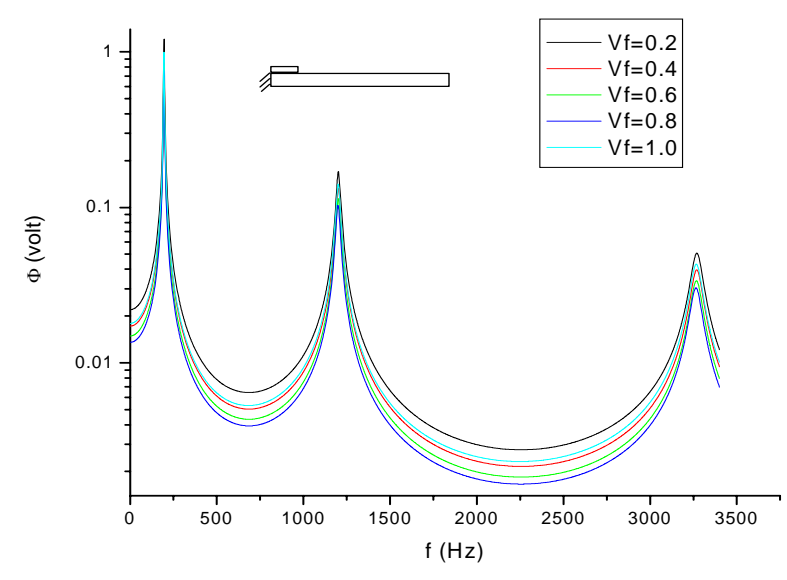

(b)

Fig 3 (a) Transverse displacement $\left(u_{z}\right)$, (b) Electric potential $(\phi)$ 


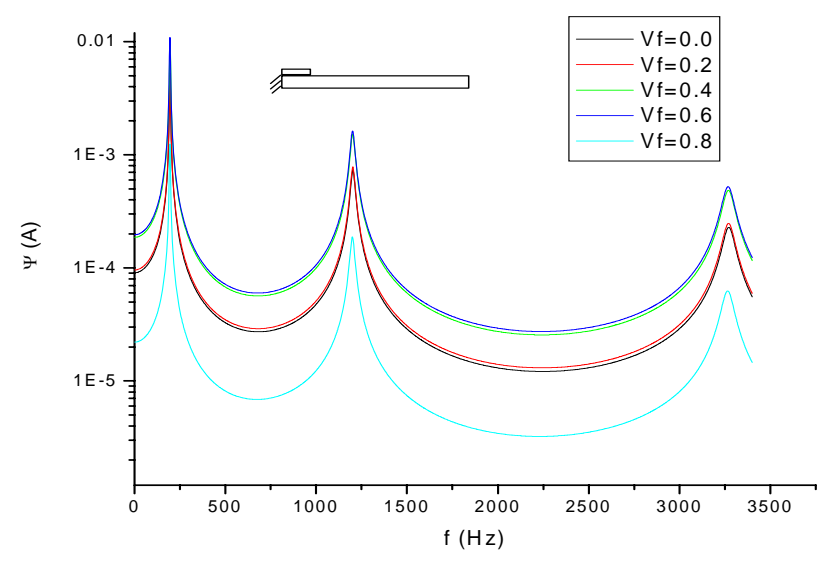

(c)

Fig 3 (c) Magnetic potential $(\psi)$ at the top surface of the MEE sensor for point load

\subsubsection{Sensor patch located at the middle}

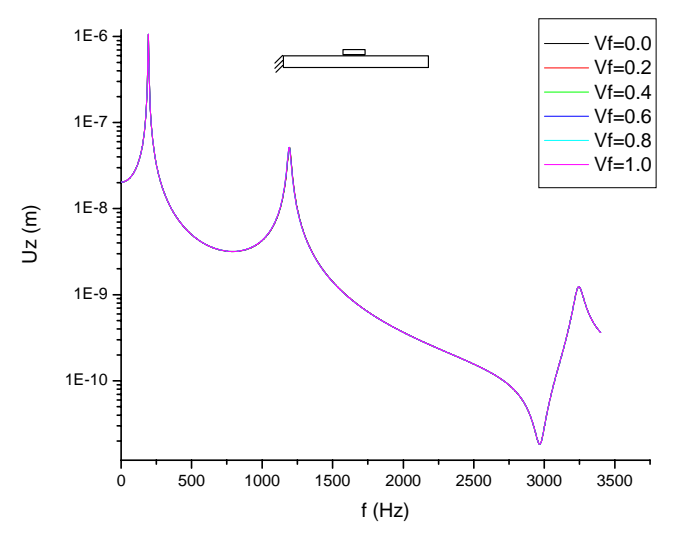

(a)

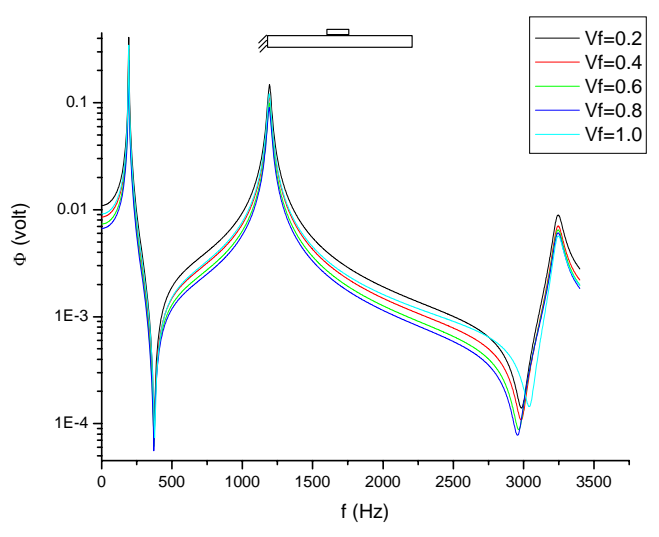

(b)

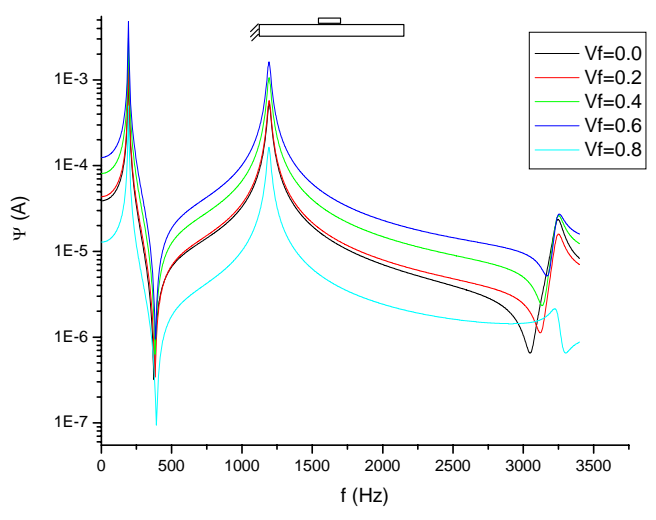

(c)

Figure 4 (a) Transverse displacement $\left(u_{z}\right)$, (b) Electric potential $(\phi)$ and (c) Magnetic potential $(\psi)$ at the top surface of the MEE sensor for point load

The transverse displacement $\left(u_{z}\right)$, electric potential $(\phi)$ and magnetic potential $(\psi)$ at the top surface of the MEE sensor when the sensor is located at the middle of the mild steel beam is shown in Figure 4. The sensor output i.e. electric potential $(\phi)$ and 
magnetic potential $(\psi)$ decreases considerably when the sensor is placed at centre. This is because the sensor is less strained as it is moved away from the clamped end. The absolute values of sensor out put during second mode of vibration of the beam are very much close to that of first mode when the sensor is placed at the middle.

5.1.3 Sensor patch located at the tip

Figure 5 shows the transverse displacement $\left(u_{z}\right)$, electric potential $(\phi)$ and magnetic potential $(\psi)$ at the top surface of the MEE sensor when the sensor is located at the free end of the mild steel beam. The third mode of vibration becomes dominant when the sensor is placed at the free end. The sensor is strained very little that the absolute values of sensor out put decreases considerably. Volume fraction 1.0 which corresponds to pure $\mathrm{BaTiO}_{3}$ gives maximum electric potential $(\phi)$. It is also seen that volume fraction 0.0 which corresponds to pure $\mathrm{CoFe}_{2} \mathrm{O}_{4}$ gives maximum magnetic potential $(\psi)$.

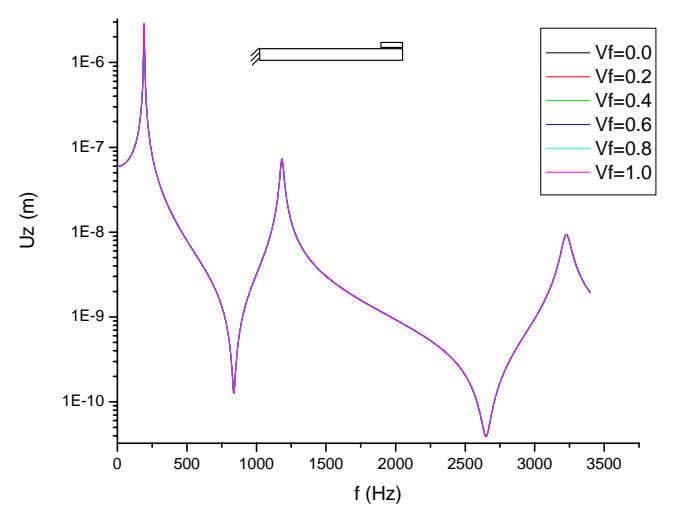

(a)

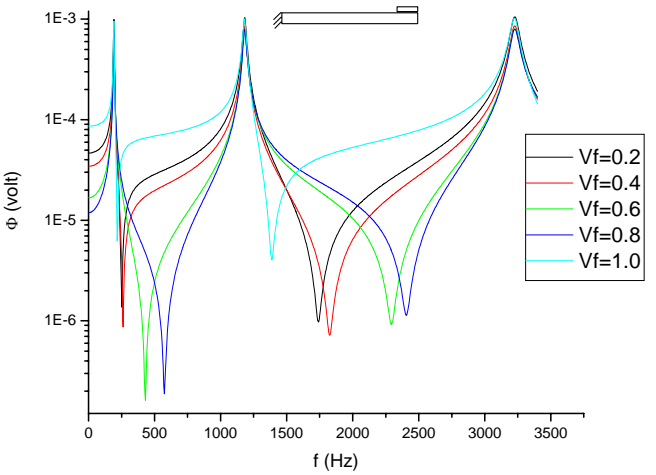

(b)

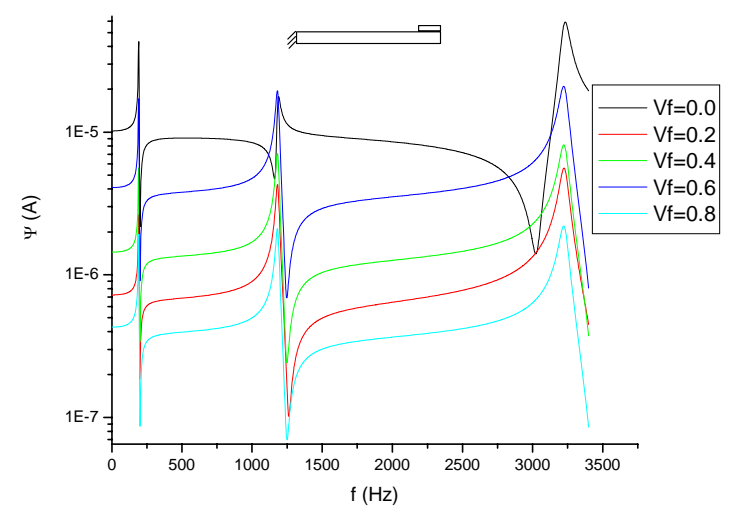

(c)

Figure 5 (a) Transverse displacement $\left(u_{z}\right)$, (b) Electric potential $(\phi)$ and (c) Magnetic potential $(\psi)$ at the top surface of the MEE sensor for point load

5.2 Uniformly distributed load at the bottom surface of the beam

The beam is clamped at one end and response of the sensor is studied when the beam is subjected to a harmonic excitation of $1 \mathrm{~N}$ in the negative $\mathrm{z}$-direction along the entire bottom surface of the beam.

5.2.1 Sensor patch located at the clamped end

Figure 6 shows the transverse displacement $\left(u_{z}\right)$, electric potential $(\phi)$ and magnetic potential $(\psi)$ at the top surface of the MEE sensor when the sensor is located at the clamped end of the mild steel beam. The sensor output i.e. electric potential $(\phi)$ and magnetic potential $(\psi)$ is high compared with point loading, as expected. It is also noticed that over a wide range of frequencies the sensor shows significantly large response when the applied load is uniformly distributed compared to point load. 


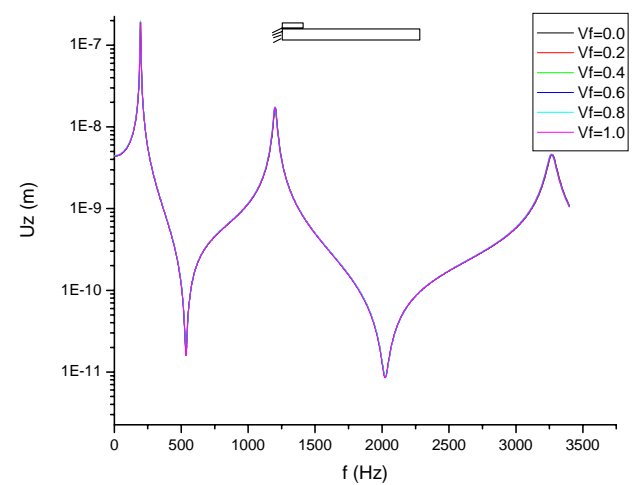

(a)

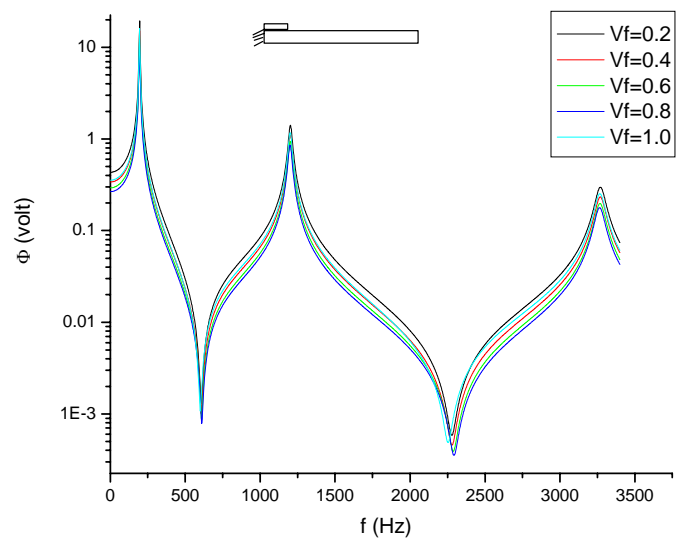

(b)

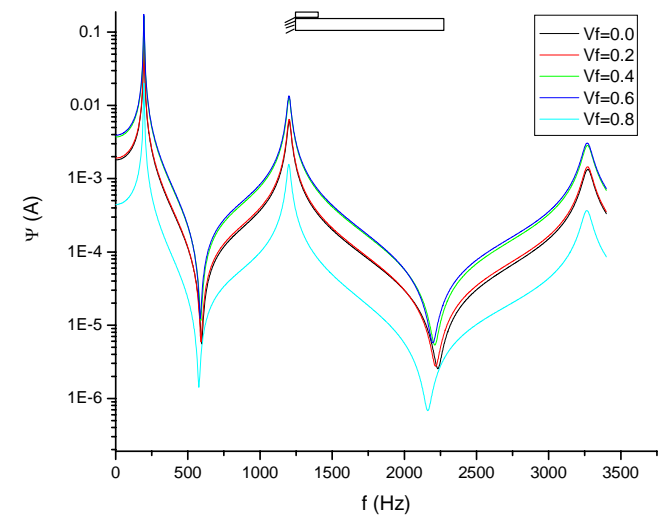

(c)

Fig 6 (a) Transverse displacement $\left(u_{z}\right)$, (b) Electric potential $(\phi)$ and (c) Magnetic potential $(\psi)$ at the top surface of the MEE sensor for UDL

\subsubsection{Sensor patch located at the middle}

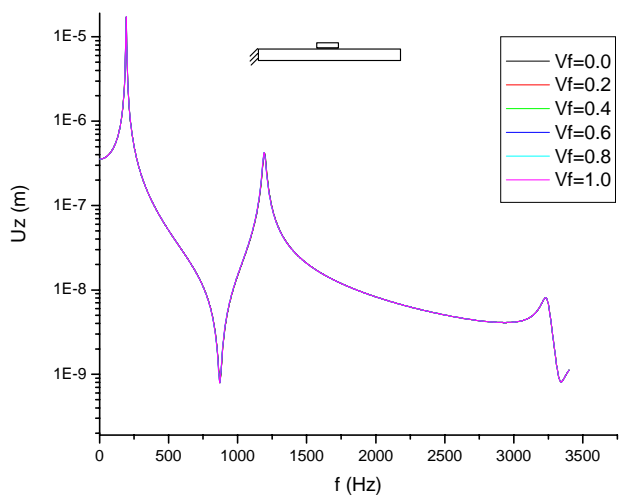

(a)

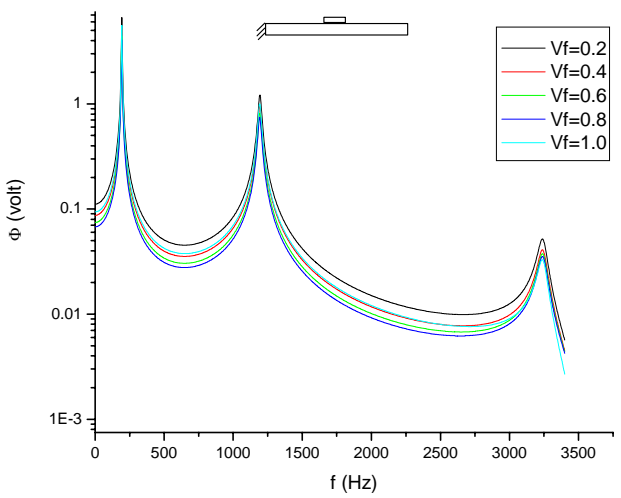

(b)

Figure 7 (a) Transverse displacement $\left(u_{z}\right)$, (b) Electric potential $(\phi)$ 


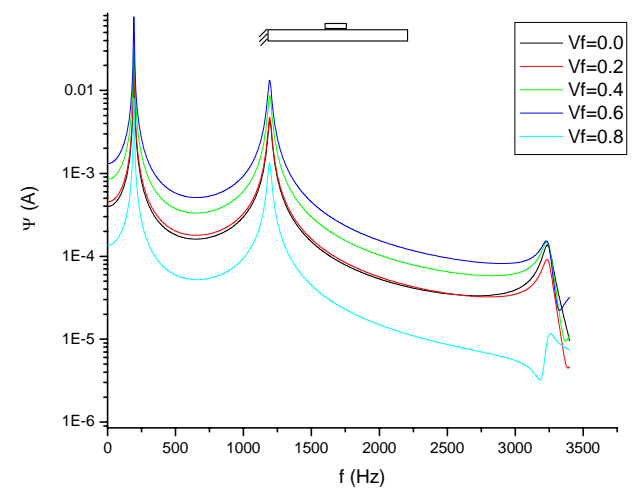

(c)

Figure 7 (c) Magnetic potential $(\psi)$ at the top surface of the MEE sensor for UDL

The transverse displacement $\left(u_{z}\right)$, electric potential $(\phi)$ and magnetic potential $(\psi)$ at the top surface of the MEE sensor when the sensor is located at the middle of the mild steel beam is shown in Figure 7. The second mode domination for response is less when the sensor is placed at the middle and the applied load is distributed uniformly. This is because strains are developed in the sensor during first mode of vibration also. The magnetic potential $(\psi)$ response become out of phase during the third mode.

5.2.3 Sensor patch located at the tip

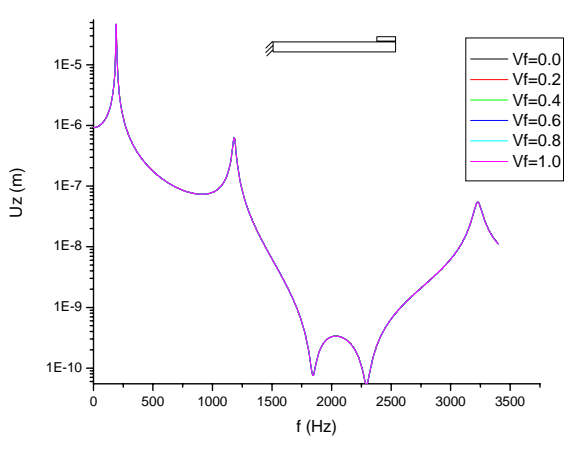

(a)

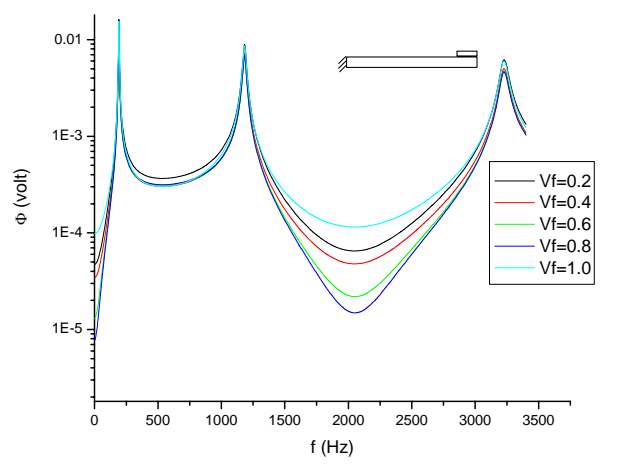

(b)

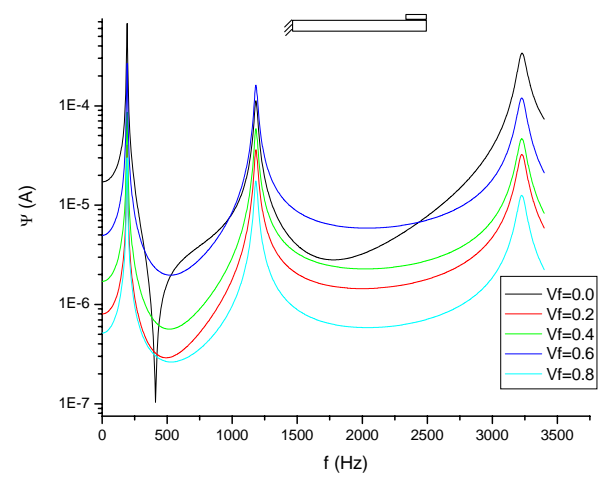

(c)

Fig 8 (a) Transverse displacement $\left(u_{z}\right)$, (b) Electric potential $(\phi)$ and (c) Magnetic potential $(\psi)$ at the top surface of the MEE sensor for UDL

Figure 8 shows the transverse displacement $\left(u_{z}\right)$, electric potential $(\phi)$ and magnetic potential $(\psi)$ at the top surface of the MEE sensor when the sensor is located at the free end of the mild steel beam. When the sensor is placed at the free end, $v_{f} 1.0$ 
which corresponds to pure $\mathrm{BaTiO}_{3}$ gives maximum electric potential $(\phi)$ and volume fraction 0.0 which corresponds to pure $\mathrm{CoFe}_{2} \mathrm{O}_{4}$ gives maximum magnetic potential $(\psi)$.

\section{Conclusions}

The finite element formulation for coupled magneto-electro-elastic sensor bonded to a mild steel beam with plane stress assumption is presented in this paper. The location of the sensor is varied along the length of the beam to study the effect on sensor response. Two types of harmonic loading i.e. a point load and a uniformly distributed load is applied harmonically to observe the sensor response at various locations.

The sensor out put viz. electric potential $(\phi)$ and magnetic potential $(\psi)$ reduces considerably as the sensor is placed away from the fixed end. This is due to the fact that the first natural frequency is the dominant mode and the beam is strained maximum at the fixed end. The electric potential $(\phi)$ is found maximum for $0.2 v_{f}$ of the composite where as the magnetic potential $(\psi)$ is found maximum for $0.6 v_{f}$ of the composite. The electric and magnetic potential decreases at higher modes. It is beneficial to place the sensor at the fixed end because it will be protected from damage as well as maximum sensor out put will be obtained. When the sensor is placed at the free end of the cantilever beam, the third mode dominates the response of the sensor.

\section{References}

Aboudi J., 2001. Micromechanical analysis of fully coupled electro-magneto-thermo-elastic multiphase composites, Smart Materials and Structures, Vol. 10, pp. 867-877.

Aimin J. and Ding H.J., 2004. Analytical solutions to magneto-electro-elastic beams, Structural Engineering and Mechanics, Vol. 18, No. 2, pp. 195-209.

Annigeri A.R., Ganesan N. and Swarnamani S., 2007. Free vibration behavior of multiphase and layered magneto-electro-elastic beams, Journal of Sound and Vibration, Vol. 299, pp. 44-63.

Atul D., Ganesan N. and Shankar K., 2009. Behaviour of magneto-electro-elastic sensors under transient mechanical loading, Sensors and Actuators A:Physical, Vol. 150, pp. 46-55.

Bakhashwain J.M., Sunar M., Hyder S.J., 2004. Finite element modeling of magnetostrictive smart structures, The Arabic journal of Science and Engineering, Vol. 29, pp. 27-138

Buchanan, G. R., 2003. Free vibration of an infinite magneto-electro-elastic cylinder, Journal of Sound and Vibration, Vol. 268, pp. 413-426.

Bhangale R.K. and Ganesan N., 2005. Free vibration studies of simply supported non-homogeneous functionally graded magneto-electro-elastic finite cylindrical shells, Journal of Sound and Vibration, Vol. 288, No. 1-2, pp. 412-422.

Buchanan G.R., 2004. Layered versus multiphase magneto-electro-elastic composites, Composites Part B, Vol. 35, pp. 413-420.

Dai H.l. and Wang X., 2006. Magneto-thermo-electro-elastic transient response in a piezoelectric hollow cylinder subjected to complex loading, International Journal of Solids and Structures, Vol. 43, pp. 5628-5646.

Ghosh, D. P. and Gopalakrishnan S., 2005. Coupled analysis of composite laminate with embedded magnetostrictive patches Smart Materials and Structures, Vol. 14, pp. 1462-1473.

Hou P.-F. and Leung A.Y.T., 2004. The transient responses of magneto-electro-elastic hollow cylinders, Smart Material systems and Structures, Vol. 13, pp. 762-776.

Marek P., 2008. Piezoelectric control of composite plate vibration: Effect of electric potential distribution, Computers and Structures, Vol. 86, pp. 948-954.

Nocolas G., Xavier M., Frederic B. and Laurent D., 2008. IEEE transactions on Magnetics, 44(2008) 834-837.

Ramirez F., Heyliger P.R. and Pan E., 2006. Free vibration response of two-dimensional magneto-electro-elastic laminated plates. Journal of Sound and Vibration, Vol. 292, pp. 626-644.

Sirohi J. and Copra I., 2000. Fundamental understanding of piezoelectric strain sensors, Journal of Intelligent Systems and Structures, Vol. 11, pp. 246-257.

Sunar, M., Ahmed Z. Al-Garni, M. H. Ali and R. Kahraman, 2002. Finite element modeling of thermopiezomagnetic smart structures, AIAA Journal, Vol. 40, pp. 1846-1851.

Wang, X. and Z. Zhong, 2003a. A finitely long circular cylindrical shell of piezoelectric/ piezomagnetic composite under pressuring and temperature change, International Journal of Engineering Science, Vol. 41, pp. 2429-2445.

Zienkiewicz O.C., Lyness John and Owen D.R.J., 1977. Three dimensional magnetic field determination using a scalar potentialA finite element solution, IEEE transactions on Magnetics, Vol. 13, pp. 1649-1656. 


\section{Biographical notes}

B. Biju has received his B.Tech Degree in Mechanical Engineering from Mahatma Gandhi University, Kottayam, Kerala, India in 1992 and M.Tech Degree in Machine Dynamics from Indian Institute of Technology Madras, Chennai, India in 2004. He is currently a Ph.D research scholar in Machine Design Section at Indian Institute of Technology Madras, Chennai, India. His research interest includes modeling and analysis of smart materials and structures.

N. Ganesan was born in India in1945. He did his graduation in 1967, post graduation in 1971 as well as Ph.D in 1976 from Indian Institute of Technology Madras, Chennai, India. He is working as Professor in the department of Mechanical Engineering at Indian Institute of Technology Madras, India. His present fields of interest are finite element modeling of smart materials and structures, sandwich structures as well as acoustics.

K. Shankar was born in India in1967. He did his graduation in 1989 from College of Engineering, Trivandrum, India. He obtained his Ph.D in 1996 from University of Oxford, England. He is working as Associate Professor in the department of Mechanical Engineering at Indian Institute of Technology Madras, India. His present fields of interest include inverse problems in vibration, system identification and design optimization.

Received November 2009

Accepted December 2009

Final acceptance in revised form December 2009 